\title{
IMAGING WITH NON-COPLANAR DATA
}

\author{
R. A. PERLEY AND T. J. CORNWELL \\ National Radio Astronomy Observatory, Socorro, N.M.
}

\begin{abstract}
The general relation between interferometric data and sky brightness requires the use of three-dimensional Fourier transforms. Only in special cases does the standard two-dimensional Fourier relations result in images free of distortions. For the VLA, use of two-dimensional transforms introduces errors which are important at low frequencies and high resolutions. We review options and progress in solving the computational problems associated with the full solution.
\end{abstract}

The measurement equation relating observed visibility, $V(u, v, w)$, to the astronomical sky brightness, $B(\ell, m)$, is:

$$
V(u, v, w)=\iint I(\ell, m) e^{-2 \pi i\left[u \ell+v m+w\left(\sqrt{1-\ell^{2}-m^{2}}-1\right)\right]} \frac{d \ell d m}{\sqrt{1-\ell^{2}-m^{2}}} .
$$

This equation is correct for a phase tracking interferometer whose fractional bandwidth $\Delta \nu / \nu \ll 1$. The general inversion problem is to recover an estimate of the sky brightness from measurements of the visibility. Note that the visibility is a function of three baseline variables, while the sky brightness is a function of two angles. There is thus no simple two-dimensional transform relation possible between the measured and desired quantities.

An important simplification, valid for all E-W interferometers and instantaneously coplanar arrays, and usable for all arrays with a sufficiently small field of view, allows the measurement equation to be written in a simpler form:

$$
V(u, v)=\iint I(\ell, m) e^{-2 \pi i(u \ell+v m)} d \ell d m
$$

which is the well-known two-dimensional Fourier transform relation used in all interferometer imaging software. The problem in using this relation is that for a two-dimensional array such as the VLA, the relation is only approximate. For any finite field size, imaging errors will occur for sources not located on the phase tracking center. It is important to understand that the origin of the error lies in the fact that the phase of a source located away from the phase-tracking center depends on the $w$-coordinate. The error incurred by ignoring the third $(w)$ term in the phase factor of the correct expression is:

$$
\Delta \phi \sim \pi w \theta^{2} \sim \pi \theta^{2} B / \lambda
$$


where $B$ is the maximum baseline, and $\theta$ is the angular offset in radians. Note that the error depends on radial distance squared, so that for sufficiently small fields, no appreciable errors will result from use of the approximate twodimension relations which results from dropping the $w$-dependence.

For illustration, consider the VLA in its ' $\mathbf{A}$ '-configuration, and take a phase error of 1 radian. The following table gives the radius at which this error occurs on the longest baseline:

\begin{tabular}{c|r}
$\lambda$ & \multicolumn{1}{c}{$\theta$} \\
\hline $6 \mathrm{~cm}$ & 4.4 arcmin \\
$20 \mathrm{~cm}$ & 8.0 arcmin \\
$90 \mathrm{~cm}$ & $17.0 \operatorname{arcmin}$ \\
\hline
\end{tabular}

High-fidelity imaging requires $\mathrm{MUCH}$ small error tolerance, and the undistored field of view decreases as the square root of the tolerable error. A more typical maximum tolerable error of one degree results in an undistorted field radius approximately one-seventh the values listed above.

These imaging errors affect virtually all VLA imaging at low frequencies, since the noise in the image is dominated by sidelobes of background sources. This 'confusion' noise cannot be removed unless the background sources are properly imaged and deconvolved.

So how can we do this? We have already noted that the general measurement equation does not admit a simple three-dimensional transform solution between the visibility data and the two-dimensional sky. However, many years ago, Barry Clark showed that an exact 3-D transform does exist between the visibility data and an 'image cube', $F(\ell, m, n)$. The relation between the real sky, $I(\ell, m)$, and the image cube is:

$$
F(\ell, m, n)=I(\ell, m) \delta\left(n+1-\sqrt{1-\ell^{2}-m^{2}}\right) .
$$

In words, the results of a straightforward three-dimensional Fourier transform of the visibility data is to produce a three-dimensional volume, whose axes are the direction cosines $(\ell, m, n)$, and in which the sky brightness is found upon a hemisphere of unit radius, centered at $(0,0,-1)$. All other regions are empty.

The above statement holds exactly for an analytic visibility function. But real data are found only at isolated points within the $(u, v, w)$ volume. Fortunately, this practical complication can be handled in exactly the same way it is for two-dimensional imaging. Thus, the image resulting from the three-dimensional transformation of sparsely sampled data is the true brightness (lying upon the hemisphere described above) convolved (in three dimensions), with a dirty beam, which is itself the transform of the function describing the samples in the visibility volume. Furthermore, the methods used in two-dimensional imaging to remove the effects of sidelobes of the dirty image all transfer unchanged to three-dimensional imaging, except that all operations must now operate within three dimensions. Thus, the most straightforward way of properly handling the non-coplanar data problem is:

1. Grid the data in three spatial dimensions and Fourier transform.

2. Deconvolve with a 3-D algorithm, . e.g. Clean. An important extra constraint, not present in 2-D deconvolution, is that no physically meaningful brightness can lie off the unit sphere. 
3. Project the resulting image upon a flat plane. This final step is essentially a problem in cartography, similar to the problem geographers have in displaying the Earth's surface on a piece of paper.

This method has the great advantage of simplicity, which translates directly into coding simplicity. There are simple answers to most of the obvious questions: The spacing of the $n$ planes will be similar to the spacing between ' $\ell$ ' and ' $m$ ' planes, i.e. $\delta n<\lambda / 2 B_{\max }$. But the total depth of the image volume will be limited by the primary beam, so that the number of $n$ planes is $N_{n} \sim$ $\theta^{2} / 2 \times 2 B_{\max } / \lambda \sim \lambda B / D^{2}$, where $D$ is the antenna diameter. For the VLA, the required number of $n$ planes is shown in the following table:

\begin{tabular}{c|rrrr}
\multicolumn{1}{c|}{$\boldsymbol{\lambda}$} & $\mathbf{A}$ & $\mathbf{B}$ & $\mathbf{C}$ & $\mathbf{D}$ \\
\hline $4 \mathrm{~m}$ & 225 & 68 & 23 & 7 \\
$1 \mathrm{~m}$ & 56 & 17 & 6 & 2 \\
$20 \mathrm{~cm}$ & 11 & 4 & 2 & 1 \\
$6 \mathrm{~cm}$ & 4 & 2 & 1 & 1 \\
\hline \hline
\end{tabular}

We have coded this method into the 'SDE' package, available on the NRAO Convexes. In practice, we make $N_{n}$ 2-D FFTs, rather than a single 3-D FFT, since the number of $n$ planes is generally small. In this multiple 2-D approach, the data are gridded simultaneously onto the $N_{n} u, v$ planes, and phase shifted by $2 \pi n w \delta n$ radians for the $n$th plane.

Although this method is simple, it can require huge memory allocations. Roughly speaking, the data volume is proportional to the cube of the maximum baseline length (and for the VLA, the data rate in ' $A$ '-configuration can be up to $230 \mathrm{MB} / \mathrm{hr}$ ), while the required memory is roughly $10^{-4} \lambda B^{3} / D^{4}$ MBytes. The following table shows the required memory allocations in MBytes to hold the map and beam:

\begin{tabular}{c|rrrr}
$\lambda$ & $\mathbf{A}$ & $\mathbf{B}$ & $\mathbf{C}$ & $\mathbf{D}$ \\
\hline $4 \mathrm{~m}$ & 45000 & 1250 & 36 & 1 \\
$1 \mathrm{~m}$ & 11000 & 310 & 9 & .25 \\
$20 \mathrm{~cm}$ & 2200 & 60 & 2 & .125 \\
$6 \mathrm{~cm}$ & 700 & 20 & 1 & .125 \\
\hline
\end{tabular}

Of course, it isn't necessary (or feasible) to obtain all this space at once - our point here is to note the magnitude of the imaging problem when one is faced with wide-field non-coplanar data. A good reason for not attempting this straightforward solution lies in the nature of the ionosphere. It is likely that the angular coherence scale of the ionosphere is of order a degree of less, so that any attempt to form an image on a scale larger than this is futile. This means that the antenna phase calibration constants are a function of angle, as well as of antenna location and time. So while the full 3-dimensional image may correct the phase errors due to the 'depth' of the array, it cannot correct for errors induced by the ionosphere. This fact of life encourages search for a solution which incorporates multiple fields.

The polyhedron imaging method is one such approach. Recognizing that the full 3-D image is mostly imaging empty 'space', the polyhedron method 
forms $N$ subfields, each of which is two-dimensional, and which is rotated to be tangent to the image hemisphere. A straightforward analysis shows that the number of 'facets' must exceed $N>2 \lambda B / D^{2}$, or twice the number of $n$ planes in the 3-D approach. However, this method makes much smaller images, naturally allows spatially dependent phase corrections, and requires vastly less memory - approximately $1000,100,10$, and 1 MB for the VLA's A, B, C, and D configurations, respectively. The disadvantages are that each facet requires a phase-shift (to the new phase tracking center) and a recomputation of the baseline coordinates (to make the facet tangent to the sphere) before gridding, and that imaging errors will still occur near the facet interfaces.

This last problem can be eliminated through a simple extension of the polyhedron approach - multi-plane polyhedrons. Here, a few (typically 3 to 5 ) $n$-planes are formed for each direction (facet). Now the imaging errors can be completely removed, although again the cost is in increased computations.

One last approach is worthy of mention, since it brings out a point of great importance. Since the VLA is instantly coplanar to a high degree of accuracy, images made from data spanning a short time will not suffer from imaging errors. One can then conceive of forming a large number of snapshots, and deconvolving them jointly. One cannot, however, simply add them up prior to deconvolution, because the geometry is different in each - the apparent locations of sources off the phase-tracking center move as a function of time. Nor can one imagine 'stretching' the dirty images to a common geometry prior to deconvolution since this will destroy the convolution relation which holds for each separately. The only way is to jointly deconvolve the $N$ input maps and beams. It should not surprise you to learn that the number of snapshots is the same as the number of $n$ planes in the 3-D approach, so that this method has nothing in it to allow easy solution of the general problem - indeed, the process of deconvolution will dominate the cost. But, there is one significant, and probably important advantage of this method - variations in the primary beam (such as pointing errors, or illumination problems causing non-circularity of the primary beam) can be made part of the deconvolution process. Certainly these complications are important in low-frequency imaging, but whether they are important enough to force adoption of this difficult solution remains to be seen. And, it must be noted that the need to remove ionospherically-induced spatially variant antenna phases would rule out this method, unless we are ready to adopt the polyhedron approach AND snapshots jointly!

We have been testing the thick polyhedron approach using $327 \mathrm{MHz}$ data, using the Cray-2 at NCSA. We found that for B-configuration data, comprising about 1 million visibilities, with 10,000 delta-function clean components, the imaging and deconvolution process took approximately 15000 seconds on the Cray-2. Note that this is for one round of imaging/deconvolution. In general, three or more rounds of self-cal will be required before the best versions of the images will result, so that a rough estimate of computer time comes to approximately 20 hours - roughly twice the time to observe. Using the rough relation that the size of the problem scales with the cube of the maximum baseline, it is clear that general solution of the $\mathbf{A}$-configuration problem awaits larger computers than now available. 